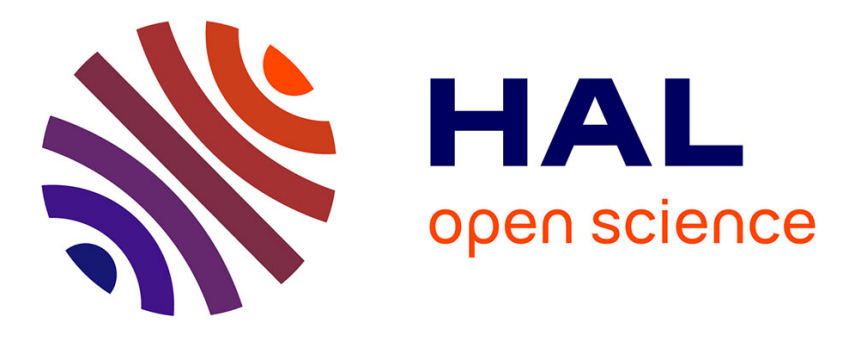

\title{
Ordinal Polymatrix Games with Incomplete Information
}

Nahla Ben Amor, Hélène Fargier, Régis Sabbadin, Meriem Trabelsi

\section{To cite this version:}

Nahla Ben Amor, Hélène Fargier, Régis Sabbadin, Meriem Trabelsi. Ordinal Polymatrix Games with Incomplete Information. 17th International Conference on Principles of Knowledge Representation and Reasoning (KR 2020), Principles of Knowledge Representation and Reasoning, Incorporated (KR, Inc.), Sep 2020, Rhodes, Greece. pp.99-108, 10.24963/kr.2020/11 . hal-02997292

\section{HAL Id: hal-02997292 \\ https://hal.science/hal-02997292}

Submitted on 10 Nov 2020

HAL is a multi-disciplinary open access archive for the deposit and dissemination of scientific research documents, whether they are published or not. The documents may come from teaching and research institutions in France or abroad, or from public or private research centers.
L'archive ouverte pluridisciplinaire HAL, est destinée au dépôt et à la diffusion de documents scientifiques de niveau recherche, publiés ou non, émanant des établissements d'enseignement et de recherche français ou étrangers, des laboratoires publics ou privés. 


\title{
Ordinal Polymatrix Games with Incomplete Information
}

\author{
Nahla Ben Amor ${ }^{1}$, Hélène Fargier ${ }^{2}$, Régis Sabbadin ${ }^{3}$, Meriem Trabelsi ${ }^{1,2}$ \\ ${ }^{1}$ LARODEC, ISG-Tunis, Tunis University, Tunisia \\ ${ }^{2}$ IRIT, Toulouse University, France \\ ${ }^{3}$ INRAE-MIAT, Toulouse University, France \\ nahla.benamor@gmx.fr, helene.fargier@irit.fr*, regis.sabbadin@inrae.fr, merieme.trabelsi@gmail.com
}

\begin{abstract}
Possibilistic games with incomplete information (П-games) constitute a suitable framework for the representation of ordinal games under incomplete knowledge. However, representing a $\Pi$-game in standard normal form requires an extensive expression of the utility functions and the possibility distribution, namely, on the product spaces of actions and types. In the present work, we propose a less costly view of $\Pi$-games, namely min-based polymatrix $\Pi$-games, which allows to concisely specify $\Pi$-games with local interactions. This framework allows, for instance, the compact representation of coordination games under uncertainty where the satisfaction of an agent is high if and only if her strategy is coherent with all of her neighbors, the game being possibly only incompletely known to the agents. Then, an important result of this paper is to show that a min-based polymatrix $\Pi$-game can be transformed, in polynomial time, into a (complete information) min-based polymatrix game with identical pure Nash equilibria. Finally, we show that the latter family of games can be solved through a MILP formulation. Experiments on variants of the GAMUT problems confirm the feasibility of this approach.
\end{abstract}

\section{Introduction}

Game theory (Morgenstern and Von Neumann 1944; Nash 1950) essentially aims at representing, explaining, and optimizing situations in which several agents, the "players", have to choose between several decisions, the "actions", the final utility of each player depending on the actions of the other players. In non-cooperative games under complete information, the players cannot coordinate their actions but each of them knows everything about the game: the players, their available actions and all their utilities.

This assumption of complete knowledge cannot always be satisfied. In the real world, players are not so well informed and have only limited knowledge about the game. This is why incomplete information games, and more particularly Bayesian games (Harsanyi 1967) have been proposed to model problems where the utility degrees are additive in essence and the knowledge of the players is quantified in a

${ }^{*}$ Helene Fargier has benefitted from the AI Interdisciplinary Institute ANITI. ANITI is funded by the French "Investing for the Future - PIA3" program under the Grant agreement n ANR-19PI3A-0004. probabilistic way. If a rich, quantifiable, information about the real world is not attainable, pure ordinal frameworks should rather be considered. This is why the original framework of ordinal games (Xu 2000; Cruz and Simaan 2000; Ouenniche, Boukouras, and Rajabi 2016) has been recently extended:

- In possibilistic Boolean games (De Clercq et al. 2018), the knowledge of each player is expressed in the framework of possibilistic logic and the players do not receive any private information before playing. Therefore, the authors consider the problem from the external point of view of an observer who proceeds to a fusion of these pieces of knowledge and computes the possibility and the necessity of a given profile of actions being a Nash equilibrium in the usual sense;

- The framework described in (Ben Amor et al. 2019a) stays at the semantic level. Nevertheless, it allows both the expression of common knowledge and the possibility for the players to get some information - captured by the notion of "type", in the same way as in Harsanyi's incomplete information games. These "possibilistic games with incomplete information" (called П-games) provide a purely ordinal notion of best response and pure equilibrium in the sense of Nash. It has been shown that any $\Pi$-game can be transformed into a standard normal form game with equivalent pure Nash equilibria (PNE) - this transformation being interesting from a descriptive point of view only, since exponentially costly in the size of the input. Notice that the input itself (the П-game) is not precisely compact: it requires an extensive expression of the utility functions and of the possibility distribution namely, on the product spaces of actions and types.

In the present work, we propose a less costly view of $\Pi-$ games. We define the framework of min-based polymatrix $\Pi$-games, which allows to concisely specify $\Pi$-games with local interactions. This framework allows, for instance, the compact representation of coordination games under possibilistic uncertainty. A motivating example, along with the basic notions on which the paper relies, is presented in Section 2. Section 3 then defines the general representation framework that we propose: min-based polymatrix $\Pi$-games, and applies it to the running example. Section 4 shows that any 2-player $\Pi$-game can be transformed into an 
equivalent min-based polymatrix game. This result is the qualitative counterpart of Howson and Rosenthals's theorem (1974) linking Bayesian games to polymatrix games. Furthermore, as soon as a simple condition on the coherence of the players' knowledge about the world is satisfied, any polymatrix $\Pi$-game can be transformed in polytime into an equivalent min-based and complete information polymatrix game. Finally, Section 5 proposes a MILP formulation of the problem of deciding whether a polymatrix $\Pi$-game admits a PNE and presents experimental results.

\section{Background}

\subsection{A Motivating Example}

Let us consider a game where several agents have to choose between multiple competing offers, e.g., choosing an internet provider. This game is a kind of "coordination game" (Simon and Wojtczak 2017). An agent is satisfied at a high degree if and only if all her neighbors choose the same service as she does - for instance, because the satisfaction of the agent is relative to the security of her communications with her neighbors and the security level of the network is not guarantee when different services are used.

This kind of game is typically based on a graph and the satisfaction of an agent is the minimum, over all her neighbors, of the satisfaction she gets in local games with single neighbor. Of course, agents may have more gradual preferences, e.g., because they prefer some providers to other ones.

The preferences of the agents may also depend on an external event, e.g., an incentive that some of them receive typically, an offer from some provider. Of course, the belief of a player about what offers her neighbors receive depends on what the player receives herself: normally, if I receive an offer, so do my neighbors; and if I do not receive anything, they do not either. But I may receive something while my neighbors do not.

In order to model such an example, we rely on two groups of works: polymatrix games on the one hand and games under incomplete information on the other hand.

\subsection{Standard Normal Form Games and Polymatrix Games}

A strategic game or standard normal form game is defined as follows:

Definition 1 (Standard normal form game (SNF)). A standard normal form game is a triple $G=\langle N, A, \mu\rangle$ where:

- $N=\{1, \ldots, n\}$ is a finite set of players;

- $A=\times_{i \in N} A_{i}$, where $A_{i}$ is a finite set of actions available to player $i . \forall a \in A, a_{i}$ is the action of $i$ in the joint action $a, a_{-i}=\left(a_{1}, \ldots, a_{i-1}, a_{i+1}, \ldots, a_{n}\right) \in$ $A_{-i}=\times_{j \neq i} A_{j}$ is its restriction to all the players but $i$ and "." denotes the concatenation, e.g., $\forall\left(a_{i}^{\prime}, a_{-i}\right)$, $a_{i}^{\prime} \cdot a_{-i}=\left(a_{1}, \ldots, a_{i-1}, a_{i}^{\prime}, a_{i+1}, \ldots, a_{n}\right)$;

- $\mu=\left\{\left(\mu_{i}\right)_{i \in N}\right\}$ is a set of real-valued utility functions: $\mu_{i}(a)$ captures the utility $i$ gets from the joint action $a$. In a SNF game, each $\mu_{i}$ is given explicitly, by a table of $|A|$ entries.
A joint (or "pure") strategy $a \in A$ is a pure Nash equilibrium (PNE) if no player can improve her utility by unilaterally changing her strategy. Formally:

Definition 2 (Pure Nash equilibrium (PNE)). A strategy profile $a \in A$ is a PNE iff: $\forall i \in N, \forall a_{i}^{\prime} \in A_{i}$ :

$$
\mu_{i}\left(a_{i} . a_{-i}\right) \geq \mu_{i}\left(a_{i}^{\prime} \cdot a_{-i}\right)
$$

Example 1. Consider a coordination game between n players, each choosing between two actions, $x$ and $y$. A player is satisfied iff all her neighbors play the same action, and is satisfied to a lower level otherwise. Of course, players may have an prior preference for $x$ or for $y$ (e.g. a preference for some provider).

Let Neigh $(i)$ denotes the set of neighbors of player $i$.

In this game $N=\{1, \ldots, n\}$ and for any $i: A_{i}=\{x, y\}$ and $\mu_{i}$ is defined as follows:

- $\mu_{i}\left(x . a_{-i}\right)=\alpha_{i, x}$ if $\forall j \in N e i g h(i), a_{i}=a_{j}=x$;

- $\mu_{i}\left(y \cdot a_{-i}\right)=\alpha_{i, y}$ if $\forall j \in N e i g h(i), a_{i}=a_{j}=y$;

- $\mu_{i}(a)=\beta$ if $\exists j \in N e i g h(i)$, s.t., $a_{i} \neq a_{j}$, i.e., when, for some $j$, players $i$ and $j$ do not coordinate.

Typically, in a coordination game, $\beta$ is lower than the $\alpha$ 's. For players who prefer to coordinate on $x$ rather than to coordinate on $y$, we have $\alpha_{i, x}>\alpha_{i, y}$ and the contrary for players who prefer a coordination on $y$ than on $x$. Table 1 presents such a game for the two players case.

Player 2

\begin{tabular}{cc|c|c|}
\multirow{2}{*}{ Player 1 } & $x$ & \multicolumn{1}{c}{$x$} & \multicolumn{1}{c}{$y$} \\
\cline { 3 - 4 } & $y$ & $\alpha_{1, x}, \alpha_{2, x}$ & $\beta, \beta$ \\
\cline { 3 - 4 } & & $\beta, \beta$ & $\alpha_{1, y}, \alpha_{2, y}$ \\
\cline { 3 - 4 } & & &
\end{tabular}

Table 1: A coordination game with two players.

When only two players are involved in $N,(x . x)$ and (y.y) are the two PNE of the game (because $\beta$ is low).

If we consider the three-player game where a central player, say player 2 , is related to the two other ones, while player 1 and 3 are related to the central player only (see the graph of Figure 1), (x.x.x) and (y.y.y) are still the only two PNE when $\beta$ is low. Suppose now that player 1 really dislikes action $x$, i.e., $\beta>\alpha_{1, x}$. Then $(x . x . x)$ is not a PNE anymore (player 1 would prefer to move to $y$ ).

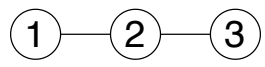

Figure 1: A graph of neighborhood between three players.

Without placing constraints on the players' utilities, describing a game in SNF involving $n$ players, each facing $d$ possible actions, requires listing $n$ utility functions of size $d^{n}$. Therefore, as mentioned by (Gottlob, Greco, and Scarcello 2005), "for large population games (modeling for instance, agents interactions over the internet), the SNF is practically unfeasible, while the more succinct graphical normal form works very well, and is actually a more natural representation". In many cases indeed, the utility of a player does not depend on the actions of all the other ones - 
the influence of what the other players decide is often local, as in our running example.

Polymatrix games (Yanovskaya 1968) have been proposed in the late 60's as a convenient way to represent games with multiple players and pairwise interactions. Polymatrix games are defined as:

Definition 3 (Polymatrix game). A polymatrix game is a four-tuple $G=\langle N, E, A, \mu\rangle$ where:

- $N=\{1, \ldots, n\}$ is a finite set of players;

- $E$ is a set of pairs of players of $N$;

- $A=\times_{i \in N} A_{i}$, where $A_{i}$ is the set of actions available to player $i$;

- $\mu=\left\{\left(\mu_{i, j}, \mu_{j, i}\right),\{i, j\} \in E\right\}$ is a set of pairs of utility functions on $A_{i} \times A_{j}: \mu_{i, j}\left(a_{i} . a_{j}\right)$ is the local utility for player $i$ of the joint action $\left(a_{i} \cdot a_{j}\right) \in A_{i} \times A_{j}$.

$(N, E)$ is a graph where nodes $N$ represent the players and edges $E$ capture the interactions between players. An absence of edge corresponds to a pair of players which utilities are independent of the actions of the other. Each edge $(i, j) \in E$ corresponds to a local 2-player game $G_{i, j}=$ $\left\langle\{i, j\}, A_{i} \times A_{j},\left\{\mu_{i, j}, \mu_{j, i}\right\}\right\rangle . G_{i, j}$ is a game in SNF, i.e., represented by two matrices - hence the name "polymatrix game".

Classical polymatrix games are sum-based: the global utility function of a player is the sum of the utilities gathered by this player in the local games she is involved in.

Definition 4 (Sum-based polymatrix game). $A$ sum-based polymatrix game is a game $G=\langle N, E, A, \mu\rangle$ where the utility of any player $i$ for the joint action a is defined as:

$$
\forall i \in N, \mu_{i}(a)=\sum_{j,\{i, j\} \in E} \mu_{i, j}\left(a_{i} \cdot a_{j}\right)
$$

In other terms, if $G$ is sum-based, its equivalent standard normal form is the game $\langle N, A, \mu\rangle$ where utilities are computed using Equation (2).

Polymatrix games can be much more frugal in memory space than SNF games - a polymatrix game indeed involves at most $2 \cdot n \cdot(n-1)$ utility functions of size $d^{2}(d$ being the maximum number of actions available to one player) - to be compared to the $n$ utility functions of size $d^{n}$ required by its standard normal form equivalent. For instance, in coordination games, the satisfaction of an agent may depend on the number of neighbors choosing the same provider as this agent (Simon and Wojtczak 2017). Unfortunately, since a min operation cannot be captured by a sum ( $\mathrm{min}$ is idempotent, sum is not), sum-based polymatrix games cannot capture the problem of Example 1, as soon as more than two players are involved. Thus, min-based polymatrix games have been recently proposed by (Azzabi et al. 2020).

Definition 5 (Min-based polymatrix game). $A$ min based polymatrix game is a polymatrix game $G=\langle N, E, A, \mu\rangle$ where the utility of player $i$ for the joint action a is defined as:

$$
\forall i \in N, \mu_{i}(a)=\min _{j,\{i, j\} \in E} \mu_{i, j}\left(a_{i} \cdot a_{j}\right)
$$

The $\mu_{i, j}$ functions take their value in an ordinal scale $\Delta$ Any ordered scale equipped with an order reversing operator $n$ may be used. For the sake of readability (and without any loss of generality), in the following we take $\Delta=[0,1]$ with $n(x)=1-x$.

\subsection{Games with Incomplete Information}

A game with incomplete information can be first understood as a set $S$ of states of nature, each state corresponding to a classical game. The utility of a player depends not only on the actions played but also on the real state of nature. Just before playing, each player $i$ will receive some information $\tau_{i}(s)$ (a "signal"). After having observed $\tau_{i}(s)$, player $i$ knows more about the real game, but several games may still be plausible; the player then conditions her knowledge according to the information received and decides which action to play. Notice that the players may have different information functions $\tau_{i}$, and thus will not share the same posterior knowledge on $S$. Let $\Theta_{i}$ be the possible pieces of information which can be received by player $i$ : $\Theta_{i}$ is called the set of "types" of $i$. The question is then, for each player, to determine an action for each of her types. Thus, in games with incomplete information, as defined by Harsanyi (1967), the set of states of the world is omitted and only the types are considered. $\Theta_{i}$ is the local state space for player $i$ and $\Theta=\Theta_{1} \times \cdots \times \Theta_{n}$ is the effective global state space ${ }^{1}$.

The question is then to determine which action to play for each type received: a pure strategy for player $i$ is a function $\sigma_{i}$ that maps each possible information $\theta_{i} \in \Theta_{i}$ to an action $a_{i} \in A_{i}$ and a joint strategy is a tuple $\sigma=\left(\sigma_{1}, \ldots, \sigma_{n}\right)$ of such functions. Formally:

Definition 6. A strategy is a vector $\sigma=\left(\sigma_{1}, \ldots, \sigma_{n}\right)$ of functions $\sigma_{i}: \Theta_{i} \rightarrow A_{i}$.

$\sigma_{i}\left(\theta_{i}\right)$ specifies the action that player $i$ will execute when receiving the private information $\theta_{i}$. In the following, $\Sigma_{i}$ denotes the set of all functions from $\Theta_{i}$ to $A_{i}$ and $\Sigma=$ $\Sigma_{1} \times \cdots \times \Sigma_{n}$ the set of all joint strategies. Sticking to our notation, any $\sigma \in \Sigma$ is the concatenation of $\sigma_{i}$ (the strategy of $i$ ) and $\sigma_{-i}$ (the strategies of all the players except $i$ ).

Example 2. Let us consider the coordination game with incentives (for the sake of brevity we assume that incentives concern only action $x$ ): each player has two types $r_{i}$ (" $i$ receives an incentive for $x$ ") and $\bar{r}_{i}$ (" $i$ does not receive an incentive for $x$ "), so $\Theta=\left\{r_{1}, \bar{r}_{1}\right\} \times \cdots \times\left\{r_{n}, \bar{r}_{n}\right\}$.

A possible strategy is $\sigma_{i}\left(r_{i}\right)=x ; \sigma_{i}\left(\bar{r}_{i}\right)=y$ if $\left(\alpha_{i, y}>\right.$ $\left.\alpha_{i, x}\right)$ and $\sigma_{i}\left(\bar{r}_{i}\right)=x$ otherwise: a player plays $x$ if receiving an incentive and her preferred action otherwise.

\footnotetext{
${ }^{1}$ The idea of Harsanyi when defining types was that a player's local state can encapsulate all the information to which the player has access: it contains the status of the external world that the player has observed but can also contain her introspective mental states. See (Brandenburger 1993; J. Aumann and Brandenburger 1995; Battigalli and Bonanno 1999; Brandenburger 2008; Eddie and Marciano 2015) for the links between belief states and types, and more generally for further developments about epistemic game theory. This kind of interpretation also complies with the semantics of epistemic logic (Fagin et al. 1999).
} 
In Bayesian games, the common knowledge over $\Theta$ is given by a probability distribution and each strategy is evaluated by its expected utility. If quantitative information about the real world is not attainable, the framework of possibilistic games should rather be considered: in a possibilistic game with incomplete information (П-game) (Ben Amor et al. 2019a), the common knowledge is given by a possibility distribution $\pi$ over the possible combinations of types, i.e., is a mapping from $\Theta$ to the ordered scale $\Delta$.

Possibilistic games with incomplete information (Пgames) are defined as:

Definition 7 ((Standard Normal Form ) П-game). A П-game is a tuple $\langle N, A, \Theta, \pi, \mu\rangle$ where:

- $N=\{1, \ldots, n\}$ is a finite set of players;

- $A=\times_{i \in N} A_{i}$ where $A_{i}$ is the set of actions of player $i$;

- $\Theta=\times_{i \in N} \Theta_{i}$, where $\Theta_{i}$ is the set of types of player $i$;

- $\pi: \Theta \mapsto \Delta$ is a joint possibility distribution over $\Theta$;

- $\mu=\left\{\left(\mu_{i}\right)_{i \in N}\right\}$ where $\mu_{i}: A \times \Theta \mapsto \Delta$ is the utility function of player $i$.

Recall that according to possibility theory (Zadeh 1978; Dubois and Prade 1988), a possibility distribution is a function from a universe of discourse (here, $\Theta$ ) to an ordered scale (typically, the unit interval). $\pi$ is assumed to be normalized: there is at least one totally possible state ( $s$ such that $\pi(s)=1)$. From $\pi$, one can compute the possibility $\Pi(E)$ and the necessity $N(E)$ of any event $E \subseteq S$ : $\Pi(E)=\sup _{s \in E} \pi(s)$ evaluates to what extent $E$ is consistent with the knowledge represented by $\pi$ while $N(E)=$ $1-\Pi(\bar{E})=1-\sup _{s \notin E} \pi(s)$ corresponds to the extent to which $\neg E$ is inconsistent and thus evaluates at which level $E$ is certainly implied by the knowledge. As for conditioning, П-games use Hisdal's definition (1978) in order to stick to the ordinal framework:

$$
\pi\left(\theta_{j} \mid \theta_{i}\right)=\left\{\begin{array}{lc}
1 & \text { if } \pi\left(\theta_{i} . \theta_{j}\right)=\pi\left(\theta_{i}\right) \\
\pi\left(\theta_{i} . \theta_{j}\right) & \text { otherwise. }
\end{array}\right.
$$

Example 3. In our example $\forall i \in N, A_{i}=\{x, y\}$ and $\Theta_{i}=\left\{r_{i}, \bar{r}_{i}\right\}$. The satisfaction of player $i$ is equal to $\beta$ if she does not coordinate with her neighbors. If she coordinates, her satisfaction when playing $x$ is increased to $\delta>\max \left\{\alpha_{i, x}, \alpha_{i, y}\right\}$ if she receives an incentive and remains $\alpha_{i, x}$ if not. Hence the following utility functions:

- if $\exists j \in N e i g h(i)$, s.t., $a_{i} \neq a_{j}$ then:

- $\mu_{i}(a, \theta)=\beta, \forall \theta$;

- if $\forall j \in N e i g h(i), a_{i}=a_{j}$ then:

- $\mu_{i}\left(x . a_{-i}, \bar{r}_{i} \cdot \theta_{-i}\right)=\alpha_{i, x}, \forall \theta_{-i}$;

- $\mu_{i}\left(x . a_{-i}, r_{i} \cdot \theta_{-i}\right)=\delta, \forall \theta_{-i}$;

- $\mu_{i}\left(y . a_{-i}, \theta\right)=\alpha_{i, y}, \forall \theta$.

Table 2 details the utility functions for the two players case.

There are two "normal" states in $\Theta=\left\{r_{1}, \bar{r}_{1}\right\} \times$ $\cdots \times\left\{r_{n}, \bar{r}_{n}\right\}$ : everybody receives an incentive for $x$ (state $\left.r=\left(r_{1}, r_{2}, \ldots, r_{n}\right)\right)$ and nobody receives anything (state $\left.\bar{r}=\left(\bar{r}_{1}, \bar{r}_{2}, \ldots \bar{r}_{n}\right)\right)$. Cases were only some players have an incentive are of course possible. This knowledge is captured by a joint possibility distribution on $\Theta: \pi(r)=\pi(\bar{r})=1$

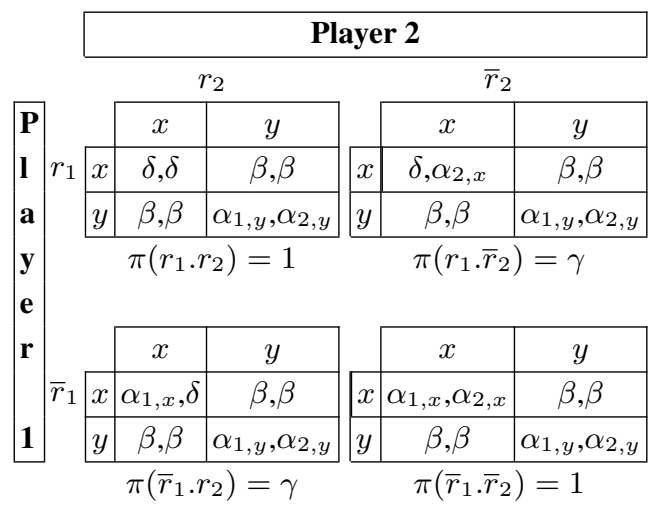

Table 2: A coordination $\Pi$-game between two players (and two types per player).

and $\pi(\theta)=\gamma$ (with $0 \leq \gamma \leq 1$ for other combinations of types $\theta \in \Theta \backslash\{r, \bar{r}\}$.

If player $i$ receives an incentive, she conditions her knowledge and we get: $\pi\left(r_{-i} \mid r_{i}\right)=1$ and $\pi\left(\theta_{-i} \mid r_{i}\right)=\gamma$ if $\theta_{-i} \neq$ $r_{-i}$, i.e., she rather believes that her neighbors also receive an incentive. Symmetrically, when she does not receive an incentive, we get: $\pi\left(\bar{r}_{-i} \mid \bar{r}_{i}\right)=1$ and $\pi\left(\theta_{-i} \mid \bar{r}_{i}\right)=\gamma$ if $\theta_{-i} \neq \bar{r}_{-i}$.

Considering qualitative (possibilistic) problems of decision under uncertainty, qualitative decision theory (Dubois and Prade 1995; Dubois, Prade, and Sabbadin 2001) proposes to evaluate the satisfaction of a player on the totally ordered scale $\Delta$ (the higher $\mu_{i}(a, \theta)$ (resp. $\pi(\theta)$ ), the more satisfied player $i$ (resp. the more plausible $\theta)$ ). $\mu_{i}(a, \theta)$ evaluates to what extent $i$ is satisfied by $a$ if the real situation is $\theta$. But, when receiving $\theta_{i}$ and deciding what to play, player $i$ does not know the full $\theta$. Her posterior knowledge about $\Theta_{-i}$ is the distribution $\pi\left(. \mid \theta_{i}\right)$. In other words, the utility of player $i$ of type $\theta_{i}$ for the joint action $a$ depends on $(i)$ the posterior knowledge of $i$ on the types of the other players and (ii) on the strategy $\sigma_{-i}$ of the other players. Then, the principles of qualitative decision theory yield the following definitions for the utility of joint strategies:

Definition 8 (Utility of an action / of a strategy). The utility of the action $a_{i}$ for player $i$ of type $\theta_{i}$ in the context of $\sigma_{-i}$ is:

$$
\begin{aligned}
& U_{i}^{\text {pes }}\left(a_{i}, \sigma_{-i}, \theta_{i}\right)= \\
& \min _{\theta_{-i} \in \Theta_{-i}} \max \left(1-\pi\left(\theta_{-i} \mid \theta_{i}\right), \mu_{i}\left(a_{i} \cdot \sigma_{-i}\left(\theta_{-i}\right), \theta_{i} \cdot \theta_{-i}\right)\right)
\end{aligned}
$$

The utility of strategy $\sigma$ to player $i$ of type $\theta_{i}$ is:

$$
U_{i}^{\text {pes }}\left(\sigma, \theta_{i}\right)=U_{i}^{\text {pes }}\left(\sigma_{i}\left(\theta_{i}\right), \sigma_{-i}, \theta_{i}\right)
$$

Note that $U_{i}^{p e s}\left(\sigma_{i}\left(\theta_{i}\right), \sigma_{-i}, \theta_{i}\right)$ is independent of the choices of player $i$ when her type is different from $\theta_{i}$.

When considering her possible strategies $\sigma_{i}: \Theta_{i} \mapsto A_{i}$, player $i$ aims at maximizing the response given the strategy $\sigma_{-i}$ of the other players. As in any kind of game, a pure Nash equilibrium (PNE) is a joint strategy from which no 
player $i$ will deviate unilaterally knowing $\sigma_{-i}$. In the possibilistic context, this leads to the following definition:

Definition 9 (Pure Nash equilibrium in a П-game). $\sigma$ is a pure Nash equilibrium (PNE) iff: $\forall i \in N, \forall \theta_{i} \in \Theta_{i}, \forall a_{i}^{\prime} \in$ $A_{i}$,

$$
U_{i}^{\text {pes }}\left(\sigma_{i}\left(\theta_{i}\right), \sigma_{-i}, \theta_{i}\right) \geq U_{i}^{\text {pes }}\left(a_{i}^{\prime}, \sigma_{-i}, \theta_{i}\right)
$$

Example 4. In the small three-player game (based on the graph of Figure 1), the equilibria are the following:

- The pure joint strategy $\sigma^{x}=x$ (every player unconditionally plays $x$ ) is a PNE when $\beta \leq$ $\min \left\{\delta, \alpha_{1, x}, \alpha_{2, x}, \alpha_{3, x}\right\}$.

- The pure joint strategy $\sigma^{y}=y$ (every player unconditionally plays $y$ ) is a PNE when $\beta \leq$ $\min \left\{\left\{\alpha_{i, x}, \alpha_{i, y}\right\}_{i=1,2,3}\right\}$.

- The pure joint strategy $\sigma$ such that any player $i$ plays $\sigma_{i}\left(r_{i}\right)=x$ and $\sigma_{i}\left(\bar{r}_{i}\right)=y$ is also a PNE, when $\gamma$ is low $\left(1-\gamma \geq \max \left\{\beta, \alpha_{i, x}, \alpha_{i, y}\right\}_{i=1,2,3}\right)$ and $\beta$ is low $\left(\beta \leq \min \left\{\delta, \alpha_{1, y}, \alpha_{2, y}, \alpha_{3, y}\right\}\right)$.

Notice that when $\gamma$ and $\beta$ increase (knowledge tends to total ignorance), there will be a point where the above third strategy will not be an equilibrium anymore.

\section{Polymatrix П-games}

The normal form representation of an incomplete information game with $n$ players, $t$ types and $d$ actions per player is very costly ( $n$ utility functions of size $t^{n} \cdot d^{n}$ and a distribution over $\Theta$, i.e., of size $t^{n}$ ) even when the problem involves local interactions only. In our example, the type vector is fixed, the satisfaction of one player is the minimum of what this player gets in a series of two-player games as the one presented in Table 1 . In order to efficiently represent such games, we now define polymatrix $\Pi$-games as min-based polymatrix games where each local game is a П-game. Such a game can be much more compact than the equivalent SNF П-game.

Definition 10 (Polymatrix П-game). A polymatrix П-game is a tuple $G=\langle N, E, A, \Theta, \mu, \pi\rangle$ where:

- $N=\{1, \ldots, n\}$ is the set of players;

- $E$ is a set of pairs of players of $N$;

- $A=\times_{i \in N} A_{i}$, where $A_{i}$ is the set of actions of player $i$;

- $\Theta=\times_{i \in N} \Theta_{i}$, where $\Theta_{i}$ is the set of types of player $i$;

- $\mu=\left\{\left(\mu_{i, j}, \mu_{j, i}\right),\{i, j\} \in E\right\}$, a set of pairs of utility functions on $A_{i} \times A_{j} \times \Theta_{i} \times \Theta_{j}$ taking their values in $\Delta$;

- $\pi=\left\{\pi_{i, j}: \theta_{i} \times \theta_{j} \mapsto \Delta,\{i, j\} \in E\right\}$ a set of pairwise possibility distributions on the $\Theta_{i} \times \Theta_{j}$ product sets.

In other terms, a polymatrix $\Pi$-game is a polymatrix game where each local game is a $\Pi$-game $\left\langle\{i, j\}, A_{i} \times A_{j}, \Theta_{i} \times\right.$ $\left.\Theta_{j}, \pi_{i, j},\left\{\mu_{i, j}, \mu_{j, i}\right\}\right\rangle$.

The condition of "common knowledge" is less natural in the present context of a series of local games than in SNF חgames - here, we assume that the knowledge of each local $\Pi$-game is common to the two players involved in, but not to the full community of players. Each player is "myopic" and her knowledge is restricted to what she knows about her neighborhood. The knowledge of player $i$ about the configurations of types of the global incomplete information game is:

$$
\pi_{i}(\theta)=\min _{j,\{i, j\} \in E} \pi_{i, j}\left(\theta_{i} \cdot \theta_{j}\right)
$$

We thus replace the condition of "common knowledge" by a condition of "coherent knowledge": there should be a $\pi$ on $\Theta$ from which the $\pi_{i, j}$ 's derive:

Assumption 1. $\exists \pi: \Theta \mapsto \Delta$ such that

$$
\forall i, j \in N, \pi_{i, j}\left(\theta_{i} \cdot \theta_{j}\right)=\underset{\theta_{-\{i, j\}}}{\max } \pi\left(\theta_{i} \cdot \theta_{j} \cdot \theta_{-\{i, j\}}\right)
$$

$\pi$ is unknown, but one knows that $\pi_{i, j}\left(\theta_{i} \cdot \theta_{j}\right)=$ $\max _{\theta_{-\{i, j\}}} \pi\left(\theta_{i} \cdot \theta_{j} \cdot \theta_{-\{i, j\}}\right)$.

Let us now study the global utility functions of the players. Each $\theta$ defines a min-based polymatrix game. The global utility of player $i$ for the joint action $a$ when the configuration of types is $\theta$ is thus:

Definition 11 (Global utility in a polymatrix П-game).

$$
\mu_{i}(a, \theta)=\min _{j,\{i, j\} \in E} \mu_{i, j}\left(a_{i} \cdot a_{j}, \theta_{i} \cdot \theta_{j}\right)
$$

If one considers all the types and the associated distribution, the polymatrix $\Pi$-game (compactly) represents the SNF II-game $\langle N, A, \Theta, \pi, \mu\rangle$. Then from the definition of the utility of a joint action/strategy for a player in a П-game (Definition 8) we have:

Definition 12 (Utility of a strategy in a polymatrix П-game).

$U_{i}^{\text {pes }}\left(\sigma, \theta_{i}\right)=\min _{\theta_{-i} \in \Theta_{-i}} \max \left(1-\pi\left(\theta_{-i} \mid \theta_{i}\right), \mu_{i}\left(\sigma\left(\theta_{i} \cdot \theta_{-i}\right), \theta\right)\right)$

Definitions 10, 11 and 12 constitute, to the best of our knowledge, the first attempt to introduce a way to cope with uncertainty in ordinal polymatrix games and more generally in ordinal graphical games.

Notice that in Definition 11, we compute, for each player and each type configuration, the utility of a player in the configuration and then compute the pessimistic utility of the player. We could have proceeded in the other way: compute the pessimistic utility in each local game and then aggregate the pessimistic utilities. The theory is fortunately sound: the two approaches (that we can call "ex-ante" and "ex-post" by reference to (Harsanyi 1967; Myerson 2004)) coincide. We can indeed prove that ${ }^{2}$

\section{Proposition 1.}

$U_{i}^{\text {pes }}\left(\sigma, \theta_{i}\right)=$
$\min _{j,\{i, j\} \in E} \min _{\theta_{j} \in \Theta_{j}} \max \left(1-\pi_{i, j}\left(\theta_{j} \mid \theta_{i}\right), \mu_{i, j}\left(\sigma\left(\theta_{i} \cdot \theta_{j}\right), \theta_{i} \cdot \theta_{j}\right)\right)$

As far as spatial complexity is concerned, it is easy to show that a polymatrix П-game can be exponentially more compact than its standard normal form equivalent. Consider our running example:

\footnotetext{
${ }^{2}$ The proofs are omitted to the sake of brevity; a version with the proof can be found at (Ben Amor et al. 2020)
} 
Example 5. The SNF П-game of Example 3 is captured by the polymatrix $\Pi$-game $G=\langle N, E, A, \Theta, \mu, \pi\rangle$ (same players, same actions and same types) where $E$ is the neighborhood relation of the original game and where the utility function $\mu_{i, j}$ of player $i$ (w.r.t. $j,(i, j) \in E$ ) is:

- $\mu_{i, j}\left(x . y, \theta_{i} \cdot \theta_{j}\right)=\mu_{i, j}\left(y \cdot x, \theta_{i} \cdot \theta_{j}\right)=\beta$;

- $\mu_{i, j}\left(x . x, r_{i} . \theta_{j}\right)=\delta$ since $\delta \geq \alpha_{i, x}, \alpha_{i, y}$;

- $\mu_{i, j}\left(x . x, \bar{r}_{i} \cdot \theta_{j}\right)=\alpha_{i, x}$

- $\mu_{i, j}\left(y \cdot y, \theta_{i} \cdot \theta_{j}\right)=\alpha_{i, y}$.

This polymatrix game contains $2 \cdot|E|$ possibility distributions of size $2 \cdot 2$, and $2 \cdot|E|$ utility functions of size $2 \cdot 2 \cdot 2 \cdot 2$, while the original SNF game involves one possibility distribution of size $2^{n}$ and $n$ utility functions of size $2^{n} \cdot 2^{n}$, whatever the connectivity of the neighborhood graph.

As far as time complexity is concerned, deciding whether a polymatrix $\Pi$-game admits a pure Nash equilibrium is an NP-hard problem because (i) any 2-player ПI-game is a (degenerated) polymatrix game and (ii) deciding whether a $\Pi-$ game admits a pure Nash equilibrium is an NP-complete problem (Ben Amor et al. 2019a). We show in the following Section that the question is "only" NP-complete. In other terms, the possible gain in compactness does not result in an increase in complexity.

\section{From Polymatrix П-games to Min-based Polymatrix Games}

In the following, we show that any polymatrix $\Pi$-game can be transformed into a min-based polymatrix game, the Nash equilibria of which are in bijection with the ones of the original game. To this extent, we first show that any 2-player $\Pi$-game can be transformed into an equivalent min-based polymatrix game - this result can be viewed as a qualitative counterpart of Howson and Rosenthals's theorem linking Bayesian games to polymatrix games (1974).

\subsection{Transforming a 2-player П-game into a Min-based Polymatrix Game}

The idea is to consider as many players as the number of pairs $\left(i, \theta_{i}\right)$, i.e., the number of players is equal to $\left|\Theta_{1}\right|+$ $\left|\Theta_{2}\right|$. Each player $\left(i, \theta_{i}\right)$ has $A_{i}$ as a set of available actions. For each joint strategy $a \in A$, the utility of player $\left(i, \theta_{i}\right)$ in the game $\left(\left(i, \theta_{i}\right),\left(j, \theta_{j}\right)\right) \in E$ in the polymatrix game is equal to the utility of the joint action $a \in A$, to player $i$ of type $\theta_{i}$ where $j$ is of type $\theta_{j}$.

Definition 13 (Polymatrix repr. of a 2-player П-game). Given a 2-player $\Pi$-game $G=\langle N=\{1,2\}, A, \Theta, \pi, \mu\rangle$, $\tilde{G}=\langle\tilde{N}, \tilde{E}, \tilde{A}, \tilde{\mu}\rangle$ is the min-based polymatrix game where:

- $\tilde{N}=\left\{\left(i, \theta_{i}\right), \forall i \in\{1,2\}, \forall \theta_{i} \in \Theta_{i}\right\}$;

- $\tilde{E}=\left\{\left(\left(i, \theta_{i}\right),\left(j, \theta_{j}\right)\right), i \neq j\right\}$;

- $\tilde{A}_{\left(i, \theta_{i}\right)}=A_{i}, \forall\left(i, \theta_{i}\right) \in \tilde{N}$;

- $\tilde{\mu}_{\left(i, \theta_{i}\right),\left(j, \theta_{j}\right)}(a)=\max \left(1-\pi\left(\theta_{j} \mid \theta_{i}\right), \mu_{i}\left(a, \theta_{i} \cdot \theta_{j}\right)\right)$, $\forall a \in \tilde{A}, \forall i, j \in N$, s.t., $i \neq j, \forall \theta_{i} \in \Theta_{i}, \forall \theta_{j} \in \Theta_{j}$.

Intuitively, each combination of types $\left(\theta_{1} \cdot \theta_{2}\right)$ in $G$ is mapped to an edge $\left(\left(1, \theta_{1}\right),\left(2, \theta_{2}\right)\right) \in E$.
Definition 14 (Transformation of a pure strategy). Let $G=$ $\langle N, A, \Theta, \pi, \mu\rangle$ be a 2-player П-game, $\tilde{G}=\langle\tilde{N}, \tilde{E}, \tilde{A}, \tilde{\mu}\rangle$ its polymatrix representation and $\sigma$ be a pure strategy in $G$.

We define $a^{\sigma}$ as the joint action in $\tilde{A}$ such that:

$$
a_{\left(i, \theta_{i}\right)}^{\sigma}=\sigma_{i}\left(\theta_{i}\right)
$$

Based on Definition 5:

$$
\tilde{\mu}_{\left(i, \theta_{i}\right)}\left(a^{\sigma}\right)=\min _{\theta_{j} \in \Theta_{j}} \tilde{\mu}_{\left(i, \theta_{i}\right),\left(j, \theta_{j}\right)}\left(a_{\left(i, \theta_{i}\right)}^{\sigma} \cdot a_{\left(j, \theta_{j}\right)}^{\sigma}\right) \text {. }
$$

We can then show that the utilities of $\sigma$ in $G$ and $a^{\sigma}$ in $\tilde{G}$ are equal:

Proposition 2. Let $G$ be a 2-player $\Pi$-game, $\tilde{G}$ its polymatrix game representation. It holds that, for any pure strategy $\sigma$ of $G: \forall i=1,2, j \neq i,\left(\theta_{i} \cdot \theta_{j}\right) \in \Theta$ :

$U_{i}^{\text {pes }}\left(\sigma, \theta_{i}\right)=\tilde{\mu}_{\left(i, \theta_{i}\right)}\left(a^{\sigma}\right)=$

$\min _{\theta_{j} \in \Theta_{j}} \tilde{\mu}_{\left(i, \theta_{i}\right),\left(j, \theta_{j}\right)}\left(a_{\left(i, \theta_{i}\right)}^{\sigma} \cdot a_{\left(j, \theta_{j}\right)}^{\sigma}\right)$.

Finally, we can show that the PNE are the same in both games:

Proposition 3. $\sigma$ is a PNE in the 2-player П-game $G=$ $\langle N, A, \Theta, \pi, \mu\rangle$ iff $a^{\sigma}$ is a PNE in its polymatrix representation $\tilde{G}=\langle\tilde{N}, \tilde{E}, \tilde{A}, \tilde{\mu}\rangle$.

Example 6. The equivalent polymatrix game $\tilde{G}$ of the two players coordination $\Pi$-game depicted in Table 2 contains four players: $\tilde{N}=\left\{\left(1, r_{1}\right),\left(1, \bar{r}_{1}\right),\left(2, r_{2}\right),\left(2, \bar{r}_{2}\right)\right\}$, all having the same set of actions $\{x, y\}$.

$\tilde{G}$ contains 4 local games (4 edges) and the utilities of each player in the equivalent game are detailed in Figure 2.
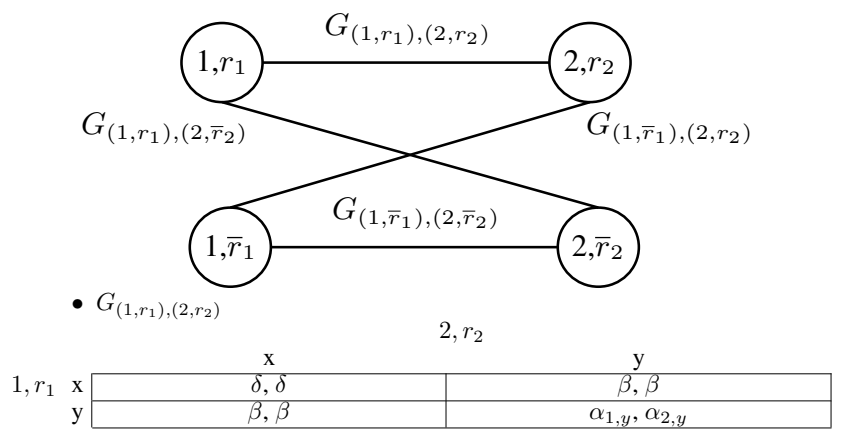

- $G_{\left(1, r_{1}\right),\left(2, \bar{r}_{2}\right)}$

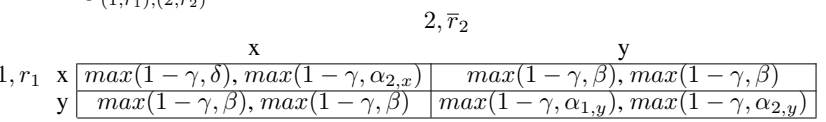

y $\max (1-\gamma, \beta), \max (1-\gamma, \beta)$
- $G_{\left(1, \bar{r}_{1}\right),\left(2, r_{2}\right)}$

$$
2, r_{2}
$$

\begin{tabular}{cc|c|c|}
\multicolumn{2}{c}{$\mathrm{x}$} & $\mathrm{y}$ \\
$1, \bar{r}_{1}$ & $\mathrm{x}$ & $\max \left(1-\gamma, \alpha_{1, x}\right), \max (1-\gamma, \delta)$ & $\max (1-\gamma, \beta), \max (1-\gamma, \beta)$ \\
\cline { 2 - 3 } & $\mathrm{y}$ & $\max (1-\gamma, \beta), \max (1-\gamma, \beta)$ & $\max \left(1-\gamma, \alpha_{1, y}\right), \max \left(1-\gamma, \alpha_{2, y}\right)$ \\
\cline { 2 - 3 } & &
\end{tabular}

- $G_{\left(1, \bar{r}_{1}\right),\left(2, \bar{r}_{2}\right)}$

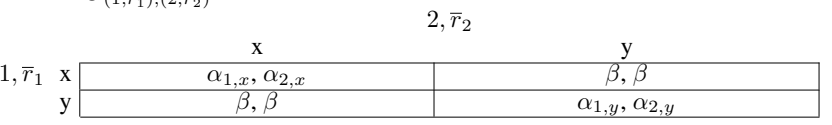

Figure 2: The polymatrix game equivalent of the 2-player П-game depicted in Table 2. 
The joint action $a^{\sigma}=(x . x . x . x)$ of $\tilde{G}$ corresponds to $\sigma^{x}$ in G. Using Equation (3), the utilities of a ${ }^{\sigma}$ in $\tilde{G}$ are:

$$
\begin{aligned}
& \mu_{\left(1, r_{1}\right)}\left(a^{\sigma}\right)=\min (\delta, \max (1-\gamma, \delta))=\delta, \\
& \mu_{\left(1, \bar{r}_{1}\right)}\left(a^{\sigma}\right)=\min \left(\max \left(1-\gamma, \alpha_{1, x}\right), \alpha_{1, x}\right)=\alpha_{1, x}, \\
& \mu_{\left(2, r_{2}\right)}\left(a^{\sigma}\right)=\min (\delta, \max (1-\gamma, \delta))=\delta, \\
& \mu_{\left(2, \bar{r}_{2}\right)}\left(a^{\sigma}\right)=\min \left(\max \left(1-\gamma, \alpha_{2, x}\right), \alpha_{2, x}\right)=\alpha_{2, x} .
\end{aligned}
$$

It can be checked that $a^{\sigma}$ is a PNE in the equivalent minbased polymatrix game.

Notice that the nodes of the polymatrix game $\tilde{G}$ represent the types in $\Theta_{1} \cup \Theta_{2}$, that the edges of $\tilde{G}$ correspond to types combinations $\theta$ in the $\Pi$-game $G$ and that the graph of $\tilde{G}$ is bipartite. More generally, the transformation of $G$ to $\tilde{G}$ is polynomial:

Proposition 4. The transformation of a 2-player $\Pi$-game $G$ into an equivalent polymatrix representation $\tilde{G}$ is in $O\left(d^{2}\right.$. $\left.t^{2}\right)$ where $t$ (resp. $d$ ) is the maximal number of types (resp. actions) per player.

Therefore, the transformation of $G$ into $\tilde{G}$ is polynomial in time and space, contrarily to the transformation into a SNF game proposed in (Ben Amor et al. 2019a).

\subsection{From polymatrix П-games to Min-based Polymatrix games with Complete Information}

When it comes to general polymatrix $\Pi$-games, we can show that polymatrix $\Pi$-games are not more expensive than classical (complete information) min-based polymatrix games. Indeed, recall that for any polymatrix $\Pi$-game, we have shown (Proposition 1) that:

$$
\begin{aligned}
& U_{i}^{\text {pes }}\left(\sigma, \theta_{i}\right)= \\
& \min _{\substack{j,\{i, j\} \in E \\
\theta_{j} \in \Theta_{j}}} \max \left(1-\pi_{i, j}\left(\theta_{j} \mid \theta_{i}\right), \mu_{i, j}\left(\sigma_{i}\left(\theta_{i}\right) \cdot \sigma_{j}\left(\theta_{j}\right), \theta_{i} \cdot \theta_{j}\right)\right)
\end{aligned}
$$

We now reuse, for every pairs of players, the transformation of the previous Section, transforming a 2-player $\Pi$ game into an equivalent min-based polymatrix game:

Definition 15. Given a polymatrix П-game $G=$ $\langle N, E, A, \Theta, \mu, \pi\rangle, \tilde{G}=\langle\tilde{N}, \tilde{E}, \tilde{A}, \tilde{U}\rangle$ is the min-based polymatrix game where:

- $\tilde{N}=\left\{\left(i, \theta_{i}\right), \forall i \in N, \forall \theta_{i} \in \Theta_{i}\right\}$;

- $\tilde{E}=\left\{\left(\left(i, \theta_{i}\right),\left(j, \theta_{j}\right)\right), i \neq j, \theta_{i} \in \Theta_{i}, \theta_{j} \in \Theta_{j}\right\}$;

- $\tilde{A}_{\left(i, \theta_{i}\right)}=A_{i}, \forall\left(i, \theta_{i}\right) \in \tilde{N}$;

- $\tilde{U}_{\left(i, \theta_{i}\right),\left(j, \theta_{j}\right)}\left(a_{i} \cdot a_{j}\right)=$ $\max \left(1-\pi_{i, j}\left(\theta_{j} \mid \theta_{i}\right), \mu_{i}\left(a_{i} . a_{j}, \theta_{i} \cdot \theta_{j}\right)\right)$,

$\forall(i, j) \in E, \forall a_{i} . a_{j} \in A_{i} \times A_{j}, \forall \theta_{i} . \theta_{j} \in \Theta_{i} \times \Theta_{j}$.

Using this definition and Proposition 1, it follows that:

Proposition 5. Let $G$ be a n-player polymatrix $\Pi$-game, $\tilde{G}$ be the corresponding min-based polymatrix representation, $\sigma$ be a pure strategy for $G$ and $a^{\sigma}$ its transformation according to Definition 14. It holds that $\forall i \in N, \forall \theta_{i} \in \Theta_{i}$,

$$
U_{i}^{\text {pes }}\left(\sigma, \theta_{i}\right)=\min _{\substack{j,\{i, j\} \in E \\ \theta_{j} \in \Theta_{j}}}\left\{\tilde{U}_{\left(i, \theta_{i}\right),\left(j, \theta_{j}\right)}\left(a_{\left(i, \theta_{i}\right)}^{\sigma} \cdot a_{\left(j, \theta_{j}\right)}^{\sigma}\right)\right\}
$$

So, the utility of $\sigma$ in $G$ is equal to the utility of $a^{\sigma}$ in $\tilde{G}$.

Since the set of pure strategies in $G$ is bijectively related to the action set $\tilde{A}$ of $\tilde{G}$, the following proposition holds:

Proposition 6. $\sigma$ is a PNE in the n-player polymatrix $\Pi$ game $G=\langle N, E, A, \Theta, \mu, \pi\rangle$ iff $a^{\sigma}$ is a PNE in the minbased polymatrix game $\tilde{G}=\langle\tilde{N}, \tilde{E}, \tilde{A}, \tilde{U}\rangle$.

\subsection{Complexity}

We can show that the size of the transformed min-based polymatrix game $\tilde{G}$ is in $O\left(|E| \cdot d^{2} \cdot t^{2}\right)$ where $t$ (resp. $d$ ) is the maximal number of types (resp. possible actions) per player: each local $\Pi$-game (we have $|E|$ local $\Pi$-games) is transformed using the transformation described in Section 4.1, the complexity of which is in $O\left(d^{2} \cdot t^{2}\right)$. Hence, the global computation cost is $O\left(|E| \cdot d^{2} \cdot t^{2}\right)$ in time and space. Now, recall that deciding the existence of a PNE in a 2-player $\Pi$-game is a NP-hard problem (Ben Amor et al. 2019a). Because any П-game can be transformed in polytime and space into an equivalent min-based polymatrix game, we first derive that ${ }^{3}$ :

Proposition 7. Determining whether there exists a PNE in a min-based polymatrix game is NP-complete. The result holds even when the graph is bipartite.

A second consequence is that deciding whether a polymatrix П-game admits a PNE is NP-complete but not harder. The problem is NP-hard because deciding whether a 2player П-game admits a PNE is NP-complete and belongs to NP since the previous transformation allows to solve it through a polytime reduction to a polymatrix min-based game.

Proposition 8. Determining whether there exists a PNE in a polymatrix $\Pi$-game is $\mathrm{NP}$-complete.

Hence, the possible gain in compactness offered by polymatrix $\Pi$-games w.r.t. SNF $\Pi$-games come with no increase in theoretical complexity.

\section{Computing a Pure Nash Equilibrium in Min-based Polymatrix Games}

\subsection{A MILP Formulation}

The basic computational problem is the search for an equilibrium in min-based polymatrix games. Taking advantage of the efficiency of modern solvers, we propose a Mixed Integer Linear Programming (MILP) formulation of the problem.

The main decision variables are boolean variables encoding the strategy searched for: each $\sigma_{i, a_{i}}$ is a boolean variable indicating whether action $a_{i}$ is prescribed for player $i$, $\forall i \in N, \forall a_{i} \in A_{i}, \sigma_{i, a_{i}} \in\{0,1\}$.

Utilities are encoded by continuous variables (we assume $\Delta=[0,1]) . U_{i, a_{i}, j}$ is a continuous variable indicating the utility of player $i$ playing action $a_{i}$ given the strategy of player $j . \forall\{i, j\} \in E, \forall a_{i} \in A_{i}, U_{i, a_{i}, j} \in[0,1] . U_{i, a_{i}}$ is a

\footnotetext{
${ }^{3}$ The membership to NP is easy to show, by a simple loop over players, actions and neighbors.
} 
continuous variable indicating the utility of player $i$ playing action $a_{i}: \forall i \in N, \forall a_{i} \in A_{i}, U_{i, a_{i}} \in[0,1]$.

We will also use boolean variables $V_{i, a_{i}, j} \in\{0,1\}$ ( $\forall i, j \in N$, s.t., $\left.\{i, j\} \in E, \forall a_{i} \in A_{i}\right)$ to express the constraints that $U_{i, a_{i}}=\min _{j,\{i, j\} \in E} U_{i, a_{i}, j}$ (and not only lower than).

The constraints are the following:

$$
\forall i \in N, \quad \sum_{a_{i} \in A_{i}} \sigma_{i, a_{i}}=1
$$

$\forall i \in N, \forall a_{i}, a_{i}^{\prime} \in A_{i}$, s.t., $a_{i} \neq a_{i}^{\prime}$,

$$
U_{i, a_{i}}-U_{i, a_{i}^{\prime}} \geq \sigma_{i, a_{i}}-1
$$

$\forall i, j \in N$, s.t., $\{i, j\} \in E, \forall a_{i} \in A_{i}$,

$$
\begin{array}{r}
U_{i, a_{i}, j}=\sum_{a_{j} \in A_{j}} \mu_{(i, j)}\left(a_{i} \cdot a_{j}\right) \times \sigma_{j, a_{j}} \\
U_{i, a_{i}} \leq U_{i, a_{i}, j} \\
U_{i, a_{i}}+V_{i, a_{i}, j} \geq U_{i, a_{i}, j} \\
\forall i \in N, \forall a_{i} \in A_{i}, \sum_{j,\{i, j\} \in E}\left(1-V_{i, a_{i}, j}\right)=1
\end{array}
$$

Constraints (6) ensure that the strategy $\sigma$ searched for specifies exactly one action $a_{i}$ for each player $i$. Constraints (7) require that the strategy built (the $\sigma_{i, a_{i}}$ which are set to 1) is a PNE: when $\sigma_{i, a_{i}}=1$, it writes $U_{i, a_{i}} \geq U_{i, a_{i}^{\prime}}$, and thus requires that player $i$ has no incentive to deviate from $a_{i}$. When action $a_{i}$ is not chosen for player $i,\left(\sigma_{i, a_{i}}=0\right)$ the constraint is always satisfied $\left(U_{i, a_{i}}-U_{i, a_{i}^{\prime}}\right.$ is always greater than -1). Constraints (9), (10) and (11) implement Equation (3). Constraints (9) ensure that the utility of player $i$ playing $a_{i}$ is lower than the minimum of utilities in local games played with players $j \in N$, i.e., the $\mu_{(i, j)}\left(a_{i} . a_{j}\right)$. Constraints (10) and (11) ensure that $U_{i, a_{i}}$ is equal to the above minimum. Whenever $V_{i, a_{i}, j}=1$, Equation (10) holds, and Equation (11) ensures that (10) is an equality for a single $j$ (minimizing $U_{i, a_{i}, j}$ ).

Let us denote $d$ the maximal number of actions of any player in the polymatrix game and $b$ the maximal number of local games in which a player can be involved. The MILP formulation contains:

- $O(n \cdot d \cdot b)$ continuous variables $U_{i, a_{i}, j}, O(n \cdot d)$ continuous variables $U_{i, a_{i}}, O(n \cdot d)$ boolean variables $\sigma_{i, a_{i}}$ and $O(n \cdot d \cdot b)$ boolean variables $V_{i, a_{i}, j}$;

- $O(n)$ constraints (6), each involving $O(d)$ variables;

- $O\left(n \cdot d^{2}\right)$ constraints (7), each involving 3 variables;

- $O(n \cdot b \cdot d)$ constraints (8), each involving $O(d)$ variables;

- $O(n \cdot b \cdot d)$ constraints (9), each involving 2 variables;

- $O(n \cdot b \cdot d)$ constraints (10), each involving 3 variables;

- $O(n \cdot d)$ constraints (11), each involving $O(b)$ variables.

The MILP can then be easily encoded in a matrix of size $O\left(n^{2} \cdot b \cdot d^{2} \cdot(b+d)\right)$.

\begin{tabular}{lcccccccccc}
\hline$\left|\Theta_{i}\right|$ & 2 & 3 & 4 & 5 & 6 & 7 & 8 & 9 & 10 & \\
\hline \multirow{2}{*}{$\mathrm{B} O S$} & 0.12 & $\mathbf{0 . 0 2}$ & 0.15 & 0.03 & 0.22 & $\mathbf{0 . 0 5}$ & 0.22 & $\mathbf{0 . 0 8}$ & 0.26 & $\mathrm{DE}$ \\
& $\mathbf{0 . 0 2}$ & $\mathbf{0 . 0 2}$ & $\mathbf{0 . 0 3}$ & $\mathbf{0 . 0 2}$ & $\mathbf{0 . 0 4}$ & 0.06 & $\mathbf{0 . 0 6}$ & 0.10 & $\mathbf{0 . 0 9}$ & $\mathrm{PE}$ \\
\hline \multirow{2}{*}{$\mathrm{C} G$} & 0.12 & $\mathbf{0 . 0 2}$ & 0.15 & 0.04 & 0.52 & $\mathbf{0 . 7 5}$ & 1.33 & 1.52 & 2.08 & $\mathrm{DE}$ \\
& $\mathbf{0 . 0 2}$ & $\mathbf{0 . 0 2}$ & $\mathbf{0 . 0 3}$ & $\mathbf{0 . 0 3}$ & $\mathbf{0 . 3 5}$ & 0.77 & $\mathbf{1 . 1 2}$ & $\mathbf{1 . 5 0}$ & $\mathbf{1 . 8 9}$ & $\mathrm{PE}$ \\
\hline \multirow{2}{*}{$\mathrm{M} \mathrm{MEG}$} & 0.12 & $\mathbf{0 . 0 2}$ & 0.15 & $\mathbf{0 . 0 2}$ & 0.22 & $\mathbf{0 . 0 4}$ & 0.22 & $\mathbf{0 . 0 7}$ & 0.25 & $\mathrm{DE}$ \\
& $\mathbf{0 . 0 2}$ & $\mathbf{0 . 0 2}$ & $\mathbf{0 . 0 3}$ & $\mathbf{0 . 0 2}$ & $\mathbf{0 . 0 4}$ & 0.05 & $\mathbf{0 . 0 5}$ & 0.09 & $\mathbf{0 . 0 8}$ & $\mathrm{PE}$ \\
\hline
\end{tabular}

Table 3: Average execution time (s) of direct encoding (DE) and polymatrix encoding (PE), $\left|A_{i}\right|=2$.

\begin{tabular}{lcccccccccc}
\hline$\left|A_{i}\right|$ & 2 & 3 & 4 & 5 & 6 & 7 & 8 & 9 & 10 & \\
\hline$\left|\Theta_{i}\right|=4$ & $\mathbf{0 . 0 7}$ & 0.08 & 0.23 & 0.19 & 0.19 & $\mathbf{0 . 1 9}$ & 0.24 & 0.26 & 0.36 & DE \\
& $\mathbf{0 . 0 7}$ & $\mathbf{0 . 0 7}$ & $\mathbf{0 . 1 8}$ & $\mathbf{0 . 1 5}$ & $\mathbf{0 . 1 8}$ & 0.20 & $\mathbf{0 . 2 3}$ & $\mathbf{0 . 2 3}$ & $\mathbf{0 . 3 3}$ & PE \\
\hline$\left|\Theta_{i}\right|=7$ & $\mathbf{0 . 1 4}$ & 0.16 & 0.18 & 0.28 & 0.33 & $\mathbf{0 . 3 9}$ & $\mathbf{0 . 3 9}$ & 0.53 & $\mathbf{0 . 7 3}$ & $\mathrm{DE}$ \\
& $\mathbf{0 . 1 4}$ & $\mathbf{0 . 1 4}$ & $\mathbf{0 . 1 6}$ & $\mathbf{0 . 2 6}$ & $\mathbf{0 . 2 8}$ & $\mathbf{0 . 3 9}$ & 0.40 & $\mathbf{0 . 5 1}$ & 0.78 & PE \\
\hline$\left|\Theta_{i}\right|=10$ & 0.14 & 0.28 & $\mathbf{0 . 3 1}$ & 0.48 & $\mathbf{0 . 5 1}$ & $\mathbf{0 . 6 7}$ & 0.72 & $\mathbf{1 . 1 1}$ & $\mathbf{3 . 2 8}$ & $\mathrm{DE}$ \\
& $\mathbf{0 . 1 3}$ & $\mathbf{0 . 2 6}$ & $\mathbf{0 . 3 1}$ & $\mathbf{0 . 4 4}$ & $\mathbf{0 . 5 1}$ & 0.74 & $\mathbf{0 . 7 0}$ & 1.16 & 3.32 & PE \\
\hline
\end{tabular}

Table 4: Average execution time (s) of direct encoding (DE) and polymatrix encoding (PE) for MEG.

\subsection{Experiments}

The goal of the first part of the experimental study is to compare the efficiency of the polymatrix encoding (PE) of 2player П-games to the one of the direct encoding (DE) (proposed by (Ben Amor et al. 2019a)), and beyond, to prove that the resolution of polymatrix $\Pi$-games is not out of reach.

To conduct our experimental study, we have used the generator of $\Pi$-games proposed in (Ben Amor et al. 2019a). This generator, based on GAMUT (Nudelman et al. 2004), is available at (Ben Amor et al. 2019b) and is essentially based on the approach of (Ceppi, Gatti, and Basilico 2009) for the generation of Bayesian games. The П-game generator first takes as input the class of the game, the number of degrees in $\Delta$, if necessary the number of players $n$, the number of actions $\left|A_{i}\right|$ and types $\left|\Theta_{i}\right|$ of player $i$. Then, this generator uses GAMUT to generate $|\Theta|$ normal form games of the class given in input. Finally, a normalized distribution $\pi: \Theta \mapsto \Delta$ is generated.

In our experiments, we study the results of the class of coordination games available on GAMUT: Battle Of The Sexes (BoS), Collaboration Game (CG) and Minimum Effort Game (MEG) (Vaughan 2004).

We start by studying 2-player П-games. We varied, for each player, the number of types from 2 to 10 . Then, according to the game's parameters, we varied just for MEG games the number of actions of each player (since in the BoS games both players have just 2 actions and CG games the number of actions of each player is equal to the number of players in the game). Then, we fixed the number of degrees $\Delta$ to 5, i.e., $\Delta=\{0,0.25,0.5,0.75,1\}$.

Furthermore, for each combination of the parameters, we generated 100 different instances and we measured the average time necessary to get a PNE by solving the MILP proposed in (Ben Amor et al. 2019a) (we denote this approach $\mathrm{DE}$, for direct encoding) and by transforming the П-game into an equivalent min-based polymatrix game and solving the above MILP of the equivalent polymatrix game proposed in Definition 13 (we denote this approach PE, for polymatrix encoding). All experiments were conducted on an Intel Xeon E5540 processor and 64GB RAM workstation. We used CPLEX (CPLEX 2009) as a MILP solver. 


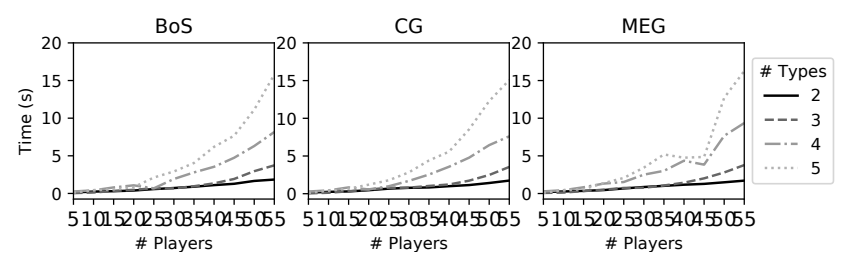

Figure 3: Average execution time (s) to find one PNE in a polymatrix $\Pi$-game, $\left|A_{i}\right|=2$.
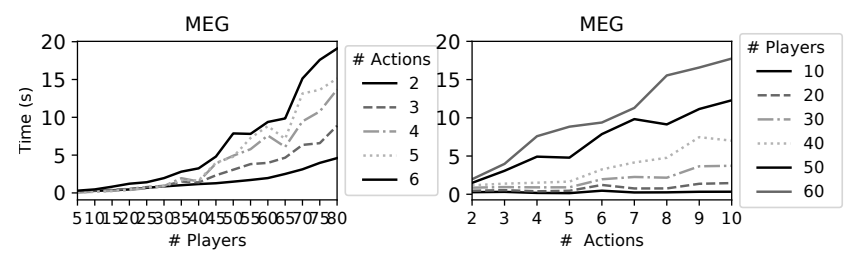

Figure 4: Average execution time (s) to find one PNE in a polymatrix $\Pi$-game, $\left|\Theta_{i}\right|=2$.

Table 3 presents the average of execution times (in seconds) needed by DE and PE to find one PNE, for 3 games classes. In the experiment reported in Table 3 , we fixed the number of actions per player to 2 , i.e., $\left|A_{i}\right|=2$ and we varied the number of types $\left|\Theta_{i}\right|$ from 2 to 10 .

Table 4 presents the average of execution times (in seconds), to find one PNE in a MEG (we tested just for MEG since the numbers of players and of actions per player in the BoS (resp. CG) game is equal to 2). We varied the number of types $\left|\Theta_{i}\right|$ from 2 to 10 and we varied the number of actions per player $\left|A_{i}\right|$ from 2 to 10 . We present the results for 3 different types' numbers (4,7 and 10).

The results show that whether we vary the number of actions or the number of types, the execution time needed to find a PNE in a 2-player $\Pi$-game using direct encoding is very close to the execution time needed to find a PNE using polymatrix encoding.

The second part of experiments was dedicated to polymatrix $\Pi$-games. We generated coordination games with different numbers of players. We varied the number of players from 5 to 80 . Then, we generated random interactions between players (ensuring that the interaction graph was connected). Then, for each edge, i.e., interaction, we generated a $\Pi$-game between 2 players using the $\Pi$-game generator proposed in (Ben Amor et al. 2019b). We varied the number of types from 2 to 9 and the number of actions (for MEG) from 2 to 10 . Then we computed the average execution time needed to find a PNE by transforming the original game into its equivalent min-based polymatrix game (using Definition 15) and solving the MILP of the latter. Notice that, the equivalent min-based polymatrix game contains $n \cdot t^{2}$ players where $t$ is the maximal number of types per player.

Figure 3 presents the average execution time needed to get one PNE in 3 different game classes. We fixed the number of actions per player to 2 .

Figures 4 (resp. 5) presents the average execution time needed to find one PNE in a MEG fixing the number of types per player to 2 (resp. the number of player to 25).
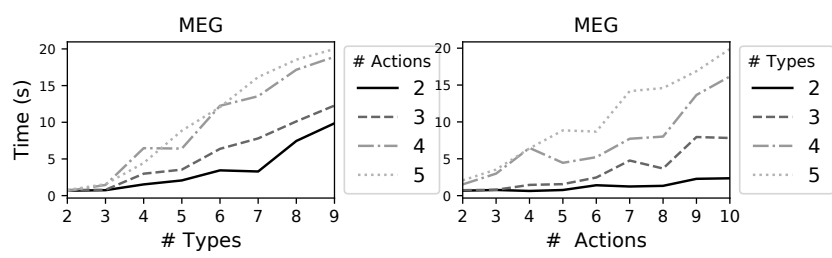

Figure 5: Average execution time (s) to find one PNE in a polymatrix $\Pi$-game, $n=25$.

Globally, MILP results confirm the feasibility of the qualitative approach of min-based polymatrix. Furthermore, the results also show that the execution time needed to solve a polymatrix $\Pi$-game increases "reasonably" (less than 20 seconds for any configuration) when increasing the number of actions or types of players.

\section{Conclusion}

The main contributions of this paper are threefold. First, we have defined a new framework for ordinal games, namely polymatrix П-games, where local games are $\Pi$-games. Such games can be exponentially more compact than the equivalent SNF П-games. Second, we have shown that any 2player $\Pi$-game can be transformed into an equivalent minbased polymatrix game, proving a qualitative counterpart of Howson and Rosenthal's theorem linking Bayesian games to polymatrix games (Howson, Joseph, and Rosenthal 1974). Then we have shown that any polymatrix $\Pi$-game can itself be transformed in polytime into an equivalent min-based polymatrix game. As a consequence, the potential gain in succinctness comes with no increase in time complexity.

We have also studied the problem of deciding whether a min-based polymatrix game admits a pure Nash equilibrium (the problem is NP-hard) and we have proposed a MILP formulation of this NP-hard problem. The experimental results confirm the theoretical ones: solving a 2-player П-game through the transformation is not more expensive than a direct solution. This underlines the importance of the basic problem, solving min-based polymatrix games.

From the point of view of knowledge representation, a straightforward extension of our work is to study, theoretically and algorithmically, the search for mixed equilibria in the sense of (Ben Amor, Fargier, and Sabbadin 2017; Hosni and Marchioni 2019). Other developments include the extension of our results to other kinds of succinct games, e.g., hypergraphical games.

Finally, in the present paper, we have suggested solving the problem through a MILP formulation, taking advantage of the available state of the art solvers. This allowed us to prove the feasibility of our approach, but a detailed algorithmic study of the direct solution of min-based polymatrix games with incomplete information is still an objective of further research. A natural extension of this paper is the development of dedicated algorithms inspired, for example, by the NashProp algorithm (Ortiz and Kearns 2003), adapted to the possibilistic framework. 


\section{Proofs}

Proof of Proposition 1. Note that

$$
\left.U_{i}^{\text {pes }}\left(\sigma, \theta_{i}\right)\right)=\min _{\theta_{-i}} \max \left(1-\pi\left(\theta_{-i} \mid \theta_{i}\right), \mu_{i}(\sigma(\theta), \theta)\right) .
$$

Now, from Definition 11, we have (slightly simplifying notations for readability):

$$
\mu_{i}(\sigma(\theta), \theta)=\min _{j,\{i, j\} \in E} \mu_{i j}\left(\sigma, \theta_{i} \cdot \theta_{j}\right) .
$$

So, we have, for all $j$ such that $\{i, j\} \in E$ :

$$
\begin{aligned}
\left.U_{i}^{\text {pes }}\left(\sigma, \theta_{i}\right)\right) \leq & \min _{\theta_{-i}} \max \left(1-\pi\left(\theta_{-i} \mid \theta_{i}\right), \mu_{i j}\left(\sigma, \theta_{i} \cdot \theta_{j}\right)\right), \\
\leq & \min _{\theta_{j}} \max \left(\min _{\theta_{-i j}}\left(1-\pi\left(\theta_{-i} \mid \theta_{i}\right)\right),\right. \\
& \left.\mu_{i j}\left(\sigma, \theta_{i} \cdot \theta_{j}\right)\right), \\
\leq & \min _{\theta_{j}} \max \left(1-\max _{\theta_{-i j}} \pi\left(\theta_{-i} \mid \theta_{i}\right)\right), \\
& \left.\mu_{i j}\left(\sigma, \theta_{i} \cdot \theta_{j}\right)\right), \\
\leq & \left.\min _{\theta_{j}} \max \left(1-\pi_{i j}\left(\theta_{j} \mid \theta_{i}\right)\right), \mu_{i j}\left(\sigma, \theta_{i} \cdot \theta_{j}\right)\right) .
\end{aligned}
$$

Thus,

$$
\begin{aligned}
\left.U_{i}^{\text {pes }}\left(\sigma, \theta_{i}\right)\right) \leq & \min _{j,\{i, j\} \in E} \min _{\theta_{j}} \max \left(1-\pi_{i j}\left(\theta_{j} \mid \theta_{i}\right)\right), \\
& \left.\mu_{i j}\left(\sigma, \theta_{i} \cdot \theta_{j}\right)\right) .
\end{aligned}
$$

Conversely, let $j^{*}$ be such that $\mu_{i}(\sigma(\theta), \theta)=$ $\mu_{i j^{*}}\left(\sigma, \theta_{i}, \theta_{j^{*}}\right)$. We have,

$$
\begin{aligned}
\left.U_{i}^{\text {pes }}\left(\sigma, \theta_{i}\right)\right)= & \min _{\theta_{-i}} \max \left(1-\pi\left(\theta_{-i} \mid \theta_{i}\right), \mu_{i j^{*}}\left(\sigma, \theta_{i} \cdot \theta_{j^{*}}\right)\right), \\
= & \min _{\theta_{j^{*}}} \max \left(\min _{\theta_{-i j^{*}}}\left(1-\pi\left(\theta_{-i} \mid \theta_{i}\right)\right),\right. \\
& \left.\mu_{i j^{*}}\left(\sigma, \theta_{i} \cdot \theta_{j^{*}}\right)\right), \\
= & \min _{\theta_{j^{*}}} \max \left(1-\max _{\theta_{-i j^{*}}} \pi\left(\theta_{-i} \mid \theta_{i}\right)\right), \\
& \left.\mu_{i j^{*}}\left(\sigma, \theta_{i} \cdot \theta_{j^{*}}\right)\right),
\end{aligned}
$$$$
\left.=\min _{\theta_{j^{*}}} \max \left(1-\pi_{i j^{*}}\left(\theta_{j^{*}} \mid \theta_{i}\right)\right), \mu_{i j^{*}}\left(\sigma, \theta_{i} \cdot \theta_{j^{*}}\right)\right)
$$

Proof of Proposition 4. Note that the space and computa-

Thus,

$$
\begin{aligned}
\left.U_{i}^{\text {pes }}\left(\sigma, \theta_{i}\right)\right) \geq & \min _{j,\{i, j\} \in E} \min _{\theta_{j}} \max \left(1-\pi_{i j}\left(\theta_{j} \mid \theta_{i}\right)\right), \\
& \left.\mu_{i j}\left(\sigma, \theta_{i} \cdot \theta_{j}\right)\right) .
\end{aligned}
$$

Proof of Proposition 2. Let us consider player 1 (the same proof holds for player 2, by symmetry). By Definition 8 , we get that: tion times of the transformation are dominated by the computation of the tables $\tilde{\mu}_{\left(i, \theta_{i}\right),\left(j, \theta_{j}\right)}(a)$. There are $2 \cdot\left|\Theta_{1}\right| \cdot\left|\Theta_{2}\right|$ such tables, each of size $\left|A_{1}\right| \cdot\left|A_{2}\right|$. The computation of $\tilde{\mu}_{\left(i, \theta_{i}\right),\left(j, \theta_{j}\right)}(a)$ for a given tuple $\left(\theta_{i}, \theta_{j}, a_{i}, a_{j}\right)$ is given in Definition 13:

$$
\tilde{\mu}_{\left(i, \theta_{i}\right),\left(j, \theta_{j}\right)}(a)=\max \left(1-\pi\left(\theta_{j} \mid \theta_{i}\right), \mu_{i}\left(a, \theta_{i} \cdot \theta_{j}\right)\right) .
$$

This computation takes constant time, so the overall time (and space) complexity is $O\left(d^{2} \cdot t^{2}\right)$.

Now, in $\tilde{G}$, we have, by definition of $\sigma=a^{\sigma}$ and of the utility of a joint strategy in a min-based polymatrix game, and the fact that player $\left(i, \theta_{i}\right)$ 's utility is independent of the

$$
\tilde{\mu}_{\left(1, \theta_{1}\right)}\left(a^{\sigma}\right)=\min _{\theta_{2} \in \Theta_{2}} \tilde{\mu}_{\left(1, \theta_{1}\right),\left(2, \theta_{2}\right)}\left(\sigma_{1}\left(\theta_{1}\right), \sigma_{2}\left(\theta_{2}\right)\right),
$$

and, from the definition of $\tilde{\mu}_{\left(1, \theta_{1}\right),\left(2, \theta_{2}\right)}$ in Definition 13:

$$
\begin{aligned}
\tilde{\mu}_{\left(1, \theta_{1}\right)}\left(a^{\sigma}\right) & =\min _{\theta_{2} \in \Theta_{2}} \max \left(1-\pi\left(\theta_{2} \mid \theta_{1}\right), \mu_{1}(\sigma(\theta), \theta)\right) \\
& =U_{1}^{\text {pes }}\left(\sigma, \theta_{1}\right) .
\end{aligned}
$$
that the utility of any pure strategy $\sigma$ in $G$ is equal to the utility of $a^{\sigma}$ in $\tilde{G}$. In order to prove the equivalence of $\sigma \rightarrow a^{\sigma}$ forms a bijection between $\Sigma=\Sigma_{1} \times \Sigma_{2}$ and $\tilde{A}$. To do so, simply note that (i) the cardinals of $\tilde{A}$ and $\Sigma$ are equal (to $\prod_{i}\left|A_{i}\right|^{\left|\Theta_{i}\right|}$ ), and (ii) the transformation is injective, i.e., if $\sigma_{i}\left(\theta_{i}\right)$ differs from $\sigma_{i}^{\prime}\left(\theta_{i}\right)$ for any $\left(i, \theta_{i}\right)$, then the pure strategies $a^{\sigma}$ and $a^{\sigma^{\prime}}$ will differ in their components $a_{\left(i, \theta_{i}\right)}^{\sigma} \neq a_{\left(i, \theta_{i}\right)}^{\sigma^{\prime}}$. As a result, the transformation is bijective, and since utilities of strategies are preserved, Nash equilibria are identical.

Proof of Proposition 5. The proof follows immediately from Proposition 1 and Definition 15.

Proof of Proposition 6. The proof is similar to that of Proposition 3 and is based on the bijection between the actions set in $\tilde{G}$ and the pure strategies set in $G$.

The proofs of the two last propositions are immediate and sketched in the main text.
Proof of Proposition 3. We have proved in Proposition 2 


\section{References}

Azzabi, A.; Ben Amor, N.; Fargier, H.; and Sabbadin, R. 2020. Ordinal graph-based games. In International Conference on Information Processing and Management of Uncertainty in Knowledge-Based Systems, 271-285.

Battigalli, P., and Bonanno, G. 1999. Recent results on belief, knowledge and the epistemic foundations of game theory. Research in Economics 53(2):149-225.

Ben Amor, N.; Fargier, H.; Sabbadin, R.; and Trabelsi, M. 2019a. Possibilistic games with incomplete information. In Proceedings of the International Joint Conference on Artificial Intelligence, 1544-1550.

Ben Amor, N.; Fargier, H.; Sabbadin, R.; and Trabelsi, M. 2019b. The Possibilistic Games Page . https://www.irit.fr/ $\% 7 E H e l e n e . F a r g i e r / P o s s i b i l i s t i c G a m e s . h t m l$ accessed on 2019-05-19.

Ben Amor, N.; Fargier, H.; Sabbadin, R.; and Trabelsi, M. 2020. Ordinal polymatrix games with incomplete information - long version. https://www.irit.fr/publis/ADRIA/ PapersFargier/KR2020long.pdf. accessed on 2020-06-23.

Ben Amor, N.; Fargier, H.; and Sabbadin, R. 2017. Equilibria in ordinal games: A framework based on possibility theory. In Proceedings of the International Joint Conference on Artificial Intelligence, 105-111.

Brandenburger, A. 1993. Hierarchies of beliefs and common knowledge. Journal of Economic Theory 59(1):189 - 198.

Brandenburger, A. 2008. Epistemic Game Theory: An Overview. London: Palgrave Macmillan UK. 1790-1791.

Ceppi, S.; Gatti, N.; and Basilico, N. 2009. Computing bayes-nash equilibria through support enumeration methods in bayesian two-player strategic-form games. In Proceedings of the International Joint Conferences on Web Intelligence and Intelligent Agent Technologies, volume 2, 541548. IEEE.

CPLEX, I. I. 2009. V12. 1: User's manual for CPLEX. International Business Machines Corporation 46(53):157.

Cruz, J., and Simaan, M. A. 2000. Ordinal games and generalized nash and stackelberg solutions. Journal of Optimization Theory and Applications 107(2):205-222.

De Clercq, S.; Schockaert, S.; Nowé, A.; and De Cock, M. 2018. Modelling incomplete information in boolean games using possibilistic logic. International Journal of Approximate Reasoning 93:1-23.

Dubois, D., and Prade, H. 1988. Possibility theory: An approach to computerized processing of uncertainty. Plenum Press, New York.

Dubois, D., and Prade, H. 1995. Possibility theory as a basis for qualitative decision theory. In Proceedings of the International Joint Conference on Artificial Intelligence, 19241930. Springer.

Dubois, D.; Prade, H.; and Sabbadin, R. 2001. Decisiontheoretic foundations of qualitative possibility theory. European Journal of Operational Research 128(3):459-478.

Eddie, D., and Marciano, S. 2015. Epistemic Game Theory. 619 - 702. chapter 12, Elsevier.
Fagin, R.; Halpern, J. Y.; Moses, Y.; and Vardi, M. Y. 1999. Common knowledge revisited. Annals of Pure and Applied Logic 96(1-3):89-105.

Gottlob, G.; Greco, G.; and Scarcello, F. 2005. Pure nash equilibria: Hard and easy games. Journal of Artificial Intelligence Research 24:357-406.

Harsanyi, J. C. 1967. Games with incomplete information played by "Bayesian" players. Management science 14(3):159-182.

Hisdal, E. 1978. Conditional possibilities independence and noninteraction. Fuzzy Sets and Systems 1(4):283-297.

Hosni, H., and Marchioni, E. 2019. Possibilistic randomisation in strategic-form games. International Journal of Approximate Reasoning 114:204-225.

Howson, J.; Joseph, T.; and Rosenthal, R. W. 1974. Bayesian equilibria of finite two-person games with incomplete information. Management Science 21(3):313-315.

J. Aumann, R., and Brandenburger, A. 1995. Epistemic conditions for nash equilibrium. Econometrica 63(5):116180 .

Morgenstern, O., and Von Neumann, J. 1944. Theory of games and economic behavior. Princeton university press.

Myerson, R. B. 2004. Comments on "games with incomplete information played by 'bayesian'players, i-iii harsanyi's games with incoplete information". Management Science 50(12_supplement):1818-1824.

Nash, J. F. 1950. Equilibrium points in n-person games. Proceedings of the national academy of sciences 36(1):4849.

Nudelman, E.; Wortman, J.; Shoham, Y.; and LeytonBrown, K. 2004. Run the gamut: A comprehensive approach to evaluating game-theoretic algorithms. In Proceedings of the International Joint Conference on Autonomous Agents and Multiagent Systems-Volume 2, 880-887. IEEE Computer Society.

Ortiz, L. E., and Kearns, M. 2003. Nash propagation for loopy graphical games. In Advances in Neural Information Processing Systems, 15, 817-824.

Ouenniche, J.; Boukouras, A.; and Rajabi, M. 2016. An ordinal game theory approach to the analysis and selection of partners in public-private partnership projects. Journal of Optimization Theory and Applications 169(1):314-343.

Simon, S., and Wojtczak, D. 2017. Constrained pure Nash equilibria in polymatrix games. In AAAI Conference on $\mathrm{Ar}$ tificial Intelligence, 691-697.

Vaughan, J. W. 2004. Gamut user guide. http://gamut. stanford.edu/userdoc.pdf. accessed on 2019-05-19.

$\mathrm{Xu}, \mathrm{C}$. 2000. Computation of noncooperative equilibria in ordinal games. European Journal of Operational Research 122(1):115-122.

Yanovskaya, E. B. 1968. Equilibrium points in polymatrix games. Litovskii Matematicheskii Sbornik 8:381-384.

Zadeh, L. A. 1978. Fuzzy sets as a basis for a theory of possibility. Fuzzy sets and systems 1(1):3-28. 